\title{
LINEAR MEASUREMENTS OF CBCT SUPERIMPOSITION
}

\author{
Sahar Mohammed Samir*, Naglaa Fathallah Ahmed ${ }^{* *}$ and Walaa Mohammed Hamed ${ }^{* *}$
}

\begin{abstract}
Objectives: To assess accuracy and reliability of linear measurements for analysis of CBCT superimposition and determine if linear measurements are enough without requiring advanced analytical tools.
\end{abstract}

Methods: Pre and post-operative CBCT scans of 20 orthodontic cases were used for CBCT superimposition. The gold standard was linear CBCT measurements on the axial section of each separate scan (pre and post) before superimposition. Voxel based and point based superimposition were done using Dolphin 3D (Dolphin Imaging and Management, Chatsworth, California, USA), In Vivo (Anatomage, San Jose, California, USA), and Ondemand 3D (Ondemand 3D; Cybermed Co., Seoul, Korea). Analysis of the superimposition was obtained by linear measurements on axial section of the superimposed models. Results were compared to the gold standard using error assessment according to Dahlberg error, Bland \&Altman method and intra-class correlation coefficient.

Results: Dolphin side by side method showed 25.4\% relative Dahlberg error (RDE), 4.28 limit of agreement (LOA) and 0.768 intra-class correlation coefficient (ICC). In-Vivo point method showed 26.75\% RDE, 4.48 LOA and 0.87 ICC. Whereas In-Vivo volume showed 24.66\% RDE, 4.15 LOA and 0.822 ICC. On Demand showed 28.44\%, 4.78 LOA and 0.7 ICC. Intra observer reliability of all methods regardless of the observers are poor.

Conclusion: The linear measurements on axial plane of CBCT superimposed models showed $3 \mathrm{~mm}$ average error. This indicates that, the statistically high relative error is clinically accepted. However, it is not recommended to depend on linear measurements alone for analysis. So, from our results, we reported weak accuracy of the linear measurements on axial plane of CBCT superimposed models and not recommending it alone for analysis.

KEYWORDS: linear measurements, CBCT, superimposition, orthodontics, analysis

* Lecturer of Oral Radiology, Faculty of Dentistry, Ain Shams University

** Assistant Professor of Oral Radiology, Faculty of Dentistry, Ain Shams University 


\section{INTRODUCTION}

CBCT superimposition has contributed widely in the evaluation of treatment outcome of many dental procedures. Three types of CBCT superimposition were described in literature; point-based, surface-based and voxel based. Several ways are available to analyze CBCT superimposition, depending on the software used. Visual and quantitative analyzing tools may include; semitransparent overlay, color coded distance map or shape correspondence mesh. However, these advanced features may not be available in every program or may include extra payment fees to activate.

On the other hand, the quantitative CBCT software tools such as linear, angular and volumetric tools, are readily available and more easily applicable for analysis. To the best of our knowledge, the use of linear measurements in analysis of superimposition has not been thoroughly evaluated. Therefore, this study aimed to answer those questions; How accurate and reliable is linear analysis of CBCT superimposed models? Can linear analysis of CBCT superimposition give satisfactory results without the advanced analytical tools?

\section{METHODS:}

CBCT superimposed models was performed on 21 non growing patients from the orthodontic clinic of our university who needed rapid maxillary expansion (RME) treatment. Unwilling and vulnerable patients were excluded. The proposal was approved by the institutional research ethics committee. All CBCT scans were acquired using I-CAT (Imaging Sciences International, Hatfield, Pennsylvania, USA) with voxel size $0.3 \mathrm{~mm}$, exposure cycle of $17.8 \mathrm{~s}, 37.1$ mass and KVP of 120. The field of view (FOV) was $23 \mathrm{~cm}$ diameter and $17 \mathrm{~cm}$ height.

The superimposition was carried out for evaluation of orthodontic rapid maxillary expansion. Superimposition of pre-treatment (T1) and posttreatment (T2) CBCT DICOM images was done.
Pre-treatment (T1) scan was obtained before start of expansion while post-treatment (T2) scan was obtained 6 months after active expansion (retention period in which the expander is kept passive in place). Two CBCT superimposition methods were performed; voxel based and point based superimposition.

For voxel-based superimposition, all DICOM files were transferred to the data base of the following 3rd party software programs; In Vivo (Anatomage, San Jose, California, USA), and Ondemand 3D (Ondemand 3D; Cybermed Co., Seoul, Korea). For point-based method; In Vivo and Dolphin 3D (Dolphin Imaging and Management, Chatsworth, California, USA) were used.

The gold standard was calculated from 3D analysis of each CBCT image (T1 and T2) using Anatomage In Vivo Dental (Anatomage, San Jose, CA, USA). The Frankfort horizontal plane was set by tracing of Porion (upper margin of external auditory meatus) and Orbitale (the lowest point in the margin of the orbit) points bilateral then the software automatically set the plane and oriented the image according to this position. The mid sagittal plane was created by tracing of Nasion (Na: the fronto nasal suture) and Basion (Ba: the most anterior point of foramen magnum) points then the program automatically located the mid sagittal plane perpendicular to the Frankfort horizontal plane and adjusted the coordinate system for analysis. Measurements were performed by tracing of each point then the software automatically calculated its distance from the midsagittal plane (Figure 1).

Points of assessment were; the mid buccal point of canine, $2^{\text {nd }}$ premolar and $1^{\text {st }}$ molar bilaterally at the axial level of anterior cemento-enamel junction (CEJ). Mathematical calculation of the resulting measurements of $\mathrm{T} 1$ and $\mathrm{T} 2$ traced points of assessment was calculated in an excel sheet to set the gold standard for the evaluation methods.

In Vivo Dental (Anatomage, San Jose, CA, USA) version 5.2 was used at the workstation of the 
specialized imaging center. The selected DICOM volume was opened then the "superimposition" tab was selected to open the superimposition tool bar. "Volume Registration" icon from the toolbar was selected to open the Volume Registration interface. Volume of Interest "VOI" was selected by left click on any of the 2D cross-sections to place the center of the VOI at the cranial base. Drawing of VOI Box was set to the default measurements ( $x$, $\mathrm{y}, \mathrm{z})=50 \mathrm{~mm} \times 50 \mathrm{~mm} \times 40 \mathrm{~mm}$. After finishing the superimposition, the layout was adjusted to the axial section at the level of CEJ of anterior teeth to start measurements at the points of assessment.

For OnDemand 3D, version 1.0.10.5385 was used at the specialized imaging center's workstation. The patient name was selected from the data base manager " $D B M$ " from the module bar at the left column of the OnDemand3 $\mathrm{D}^{\mathrm{TM}}$ layout. To launch the "fusion" module, the two series of data (T1 and T2 images) were selected at once using the [CTRL] or [Shift] keys from DBM. The two sets of data from the "Loading Option" dialog was selected again using either "Shift" or "Select All" at once then "OK". The pre-operative image T1 was set as the adjustable one (2ry) and the post-operative T2 image was set as the (1ry) or the fixed image. The region of interest was adjusted to the anterior cranial base using "VOI" icon. "Manual Registration" tab was selected $1^{\text {st }}$ before automatic registration. The secondary image was manipulated by Click and drag to re-position it close to the primary image. "Auto Registration" module was then clicked to start voxel-based registration. The layout was set to "MPR 2x2." The viewing tools and the color scale were adjusted to get the best image quality. Direct measurement of the points of assessment were done on the axial cut at the level of CEJ of anterior teeth using (the ruler) tool on the superimposed model. The results were then saved as projects using (save project tool).

For point-based method, Number of points was set to 6 at the side tool bar. Six reference points were selected to delineate the two perpendicular planes; the Frankfort horizontal plane and the midsagittal plane.

\section{Frankfort horizontal plane:}

1. Porion $(\mathrm{P})$ : The most superior point of external auditory meatus (right and left)

2. Orbitale $(\mathrm{O})$ : The most inferior point of infraorbital margin (right and left).

\section{Midsagittal plane:}

1. Nasion $(\mathrm{Na})$ : The fronto-nasal suture

2. Basion $(\mathrm{Ba})$ : The most anterior point of foramen magnum
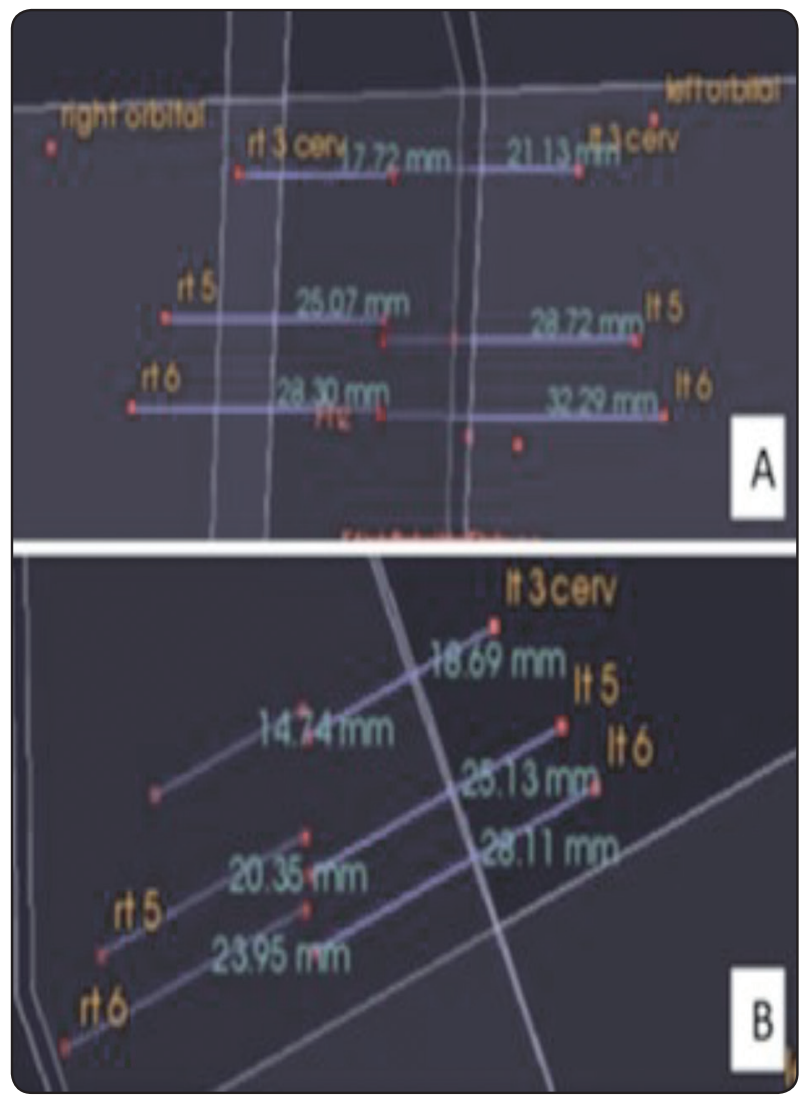

Figure 1: Setting the gold standard.

A: Post-operative measurements

B: Pre-operative measurements 
On the 3D volume, the points were located by identifying points corresponding to each other on both volumes. Image analysis of the fused model using Dolphin 3D was performed on "Analysis/ Verify results" tab. Brightness, contrast and colors were adjusted to obtain the best image quality. "Measure" module was selected to measure the points of assessment on the axial fused image. For In Vivo Dental version 5.2 was used and both volumes were aligned side by side and points were selected on each volume by pressing "space" button on the keyboard then right mouse click to finish the registration procedure. "Superimposition aligner" tab was used to fine adjust the registration. The layout was adjusted to the axial section at the level of cervical line where the points of assessment were measured using "Distance measurement" tool on the tool bar. The resulting CBCT superimposed models are illustrated in (Figure 2).

\section{RESULTS}

The study tested the accuracy of the linear measurements on the superimposed models compared to the linear measurements on the 3D traced images using error assessment of each software program according to Dahlberg error (DE), Bland \&Altman method and intra-class correlation coefficient (ICC).

Table 1 showed the error percent and the agreement between the tested methods and the gold standard. In Vivo volume method showed the best RDE and ICC (24.66\% and 0.822). While OnDemand had the worst performance $(28.44 \%$ RDE and 0.702 ICC).

Tables 2, 3 and 4, showed the inter-observer reliability. In-vivo volume method had the lowest RDE $20.9 \%$ and the highest ICC $0.915 \%$. Dolphin side by side method showed $0.502 \mathrm{~mm}$ LOA. The worst reliability was detected by high RDE of In Vivo point (37.7\%), the Lowest ICC of Dolphin side by side (0.625) and the lowest LOA of In Vivo volume (0.104). In general, Intra observer reliability of all methods regardless of the observers were inconsistent.

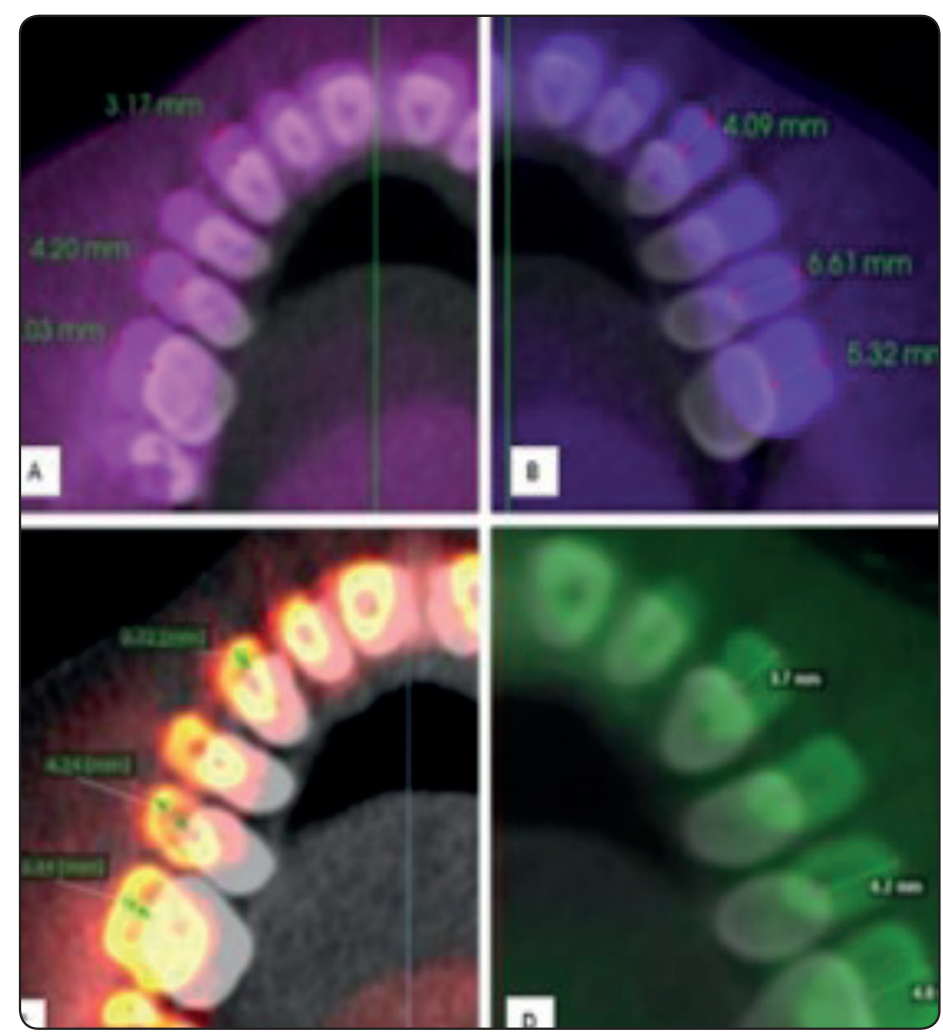

Fig. (2) Linear measurements of the superimposed images.
A: In-vivo point method
B: In-vivo volume method
C: OnDemand method
D: Dolphin side by side method 
TABLE (1) Accuracy of all superimposition methods

\begin{tabular}{|c|c|c|c|c|c|c|c|c|c|c|c|c|}
\hline \multirow{3}{*}{$\begin{array}{l}\text { Method } \\
\text { of } \\
\text { superim- } \\
\text { position }\end{array}$} & & \multirow{2}{*}{\multicolumn{3}{|c|}{$\begin{array}{c}\text { Dahlberg error } \\
\text { assessment }\end{array}$}} & \multirow{2}{*}{\multicolumn{5}{|c|}{$\begin{array}{c}\text { Bland \& Altman method } \\
95 \% \text { confidence LOA }\end{array}$}} & \multirow{2}{*}{\multicolumn{3}{|c|}{$\begin{array}{l}\text { Intraclass Correlation } \\
\text { Coefficient } \\
95 \% \text { confidence limits }\end{array}$}} \\
\hline & & & & & & & & & & & & \\
\hline & & Mean & SD & $\mathrm{DE}$ & RDE & $\begin{array}{c}\text { Mean of } \\
\text { Difference }\end{array}$ & Lower & Upper & \begin{tabular}{|l|} 
Range \\
of LOA
\end{tabular} & ICC & Lower & Upper \\
\hline \multirow{2}{*}{$\begin{array}{c}\text { Dolphin } \\
\text { side by side }\end{array}$} & Gold & 3.01 & 1.25 & \multirow{2}{*}{0.76} & \multirow{2}{*}{$25.4 \%$} & \multirow{2}{*}{0.06} & \multirow{2}{*}{-2.08} & \multirow{2}{*}{2.19} & \multirow{2}{*}{4.28} & \multirow{2}{*}{0.768} & \multirow{2}{*}{0.585} & \multirow{2}{*}{0.870} \\
\hline & Measured & 2.95 & 1.25 & & & & & & & & & \\
\hline \multirow{2}{*}{$\begin{array}{l}\text { Invivo } \\
\text { point }\end{array}$} & Gold & 3.01 & 1.25 & \multirow{2}{*}{0.81} & \multirow{2}{*}{$26.75 \%$} & \multirow{2}{*}{0.13} & \multirow{2}{*}{-2.11} & \multirow{2}{*}{2.37} & \multirow{2}{*}{4.48} & \multirow{2}{*}{0.769} & \multirow{2}{*}{0.589} & \multirow{2}{*}{0.870} \\
\hline & Measured & 2.88 & 1.38 & & & & & & & & & \\
\hline \multirow{2}{*}{$\begin{array}{l}\text { Invivo } \\
\text { Volume }\end{array}$} & Gold & 3.01 & 1.25 & \multirow{2}{*}{0.74} & \multirow{2}{*}{$24.66 \%$} & \multirow{2}{*}{-0.05} & \multirow{2}{*}{-2.13} & \multirow{2}{*}{2.03} & \multirow{2}{*}{4.15} & \multirow{2}{*}{0.822} & \multirow{2}{*}{0.682} & \multirow{2}{*}{0.900} \\
\hline & Measured & 3.06 & 1.45 & & & & & & & & & \\
\hline \multirow{2}{*}{$\begin{array}{c}\text { On } \\
\text { Demand }\end{array}$} & Gold & 3.01 & 1.25 & \multirow{2}{*}{0.86} & \multirow{2}{*}{$28.44 \%$} & 010 & & & & & & \\
\hline & Measured & 3.11 & 1.29 & & & -0.10 & $-2 .+7$ & 2.00 & 7.10 & 0.102 & 0.701 & 0.00 \\
\hline
\end{tabular}

SD: standard deviation, DE: Dahlberg error, RDE: Relative Dahlberg Error, LOA: limit of agreement, ICC: Intraclass Correlation Coefficient

TABLE (2) Inter-observer reliability between observers 1 and 2

\begin{tabular}{|c|c|c|c|c|c|c|c|c|}
\hline & \multicolumn{2}{|c|}{ Dolphin side by side } & \multicolumn{2}{|c|}{ Invivo point } & \multicolumn{2}{|c|}{ Invivo volume } & \multicolumn{2}{|c|}{ On Demand } \\
\hline & Obs 1 & Obs 2 & Obs 1 & Obs 2 & Obs 1 & Obs 2 & Obs 1 & Obs 2 \\
\hline Mean & 2.93 & 3.10 & 2.87 & 3.12 & 3.26 & 3.21 & 3.38 & 3.04 \\
\hline SD & 1.45 & 1.64 & 1.81 & 1.39 & 1.70 & 1.73 & 1.75 & 1.32 \\
\hline DE & \multicolumn{2}{|c|}{0.999} & \multicolumn{2}{|c|}{0.972} & \multicolumn{2}{|c|}{0.677} & \multicolumn{2}{|c|}{0.856} \\
\hline RDE & \multicolumn{2}{|c|}{$33.1 \%$} & \multicolumn{2}{|c|}{$32.4 \%$} & \multicolumn{2}{|c|}{$20.9 \%$} & \multicolumn{2}{|c|}{$26.7 \%$} \\
\hline ICC & \multicolumn{2}{|c|}{0.735} & \multicolumn{2}{|c|}{0.779} & \multicolumn{2}{|c|}{0.915} & \multicolumn{2}{|c|}{0.823} \\
\hline 95\% confidence lower limits & \multicolumn{2}{|c|}{0.508} & \multicolumn{2}{|c|}{0.608} & \multicolumn{2}{|c|}{0.849} & \multicolumn{2}{|c|}{0.682} \\
\hline 95\% confidence Upper limits & \multicolumn{2}{|c|}{0.857} & \multicolumn{2}{|c|}{0.876} & \multicolumn{2}{|c|}{0.952} & \multicolumn{2}{|c|}{0.901} \\
\hline LOA Range & \multicolumn{2}{|c|}{0.349} & \multicolumn{2}{|c|}{0.268} & \multicolumn{2}{|c|}{0.104} & \multicolumn{2}{|c|}{0.218} \\
\hline
\end{tabular}

TABLE (3) Inter-observer reliability between observers 1 and 3

\begin{tabular}{|c|c|c|c|c|c|c|c|c|}
\hline & \multicolumn{2}{|c|}{ Dolphin side by side } & \multicolumn{2}{|c|}{ Invivo point } & \multicolumn{2}{|c|}{ Invivo voluome } & \multicolumn{2}{|c|}{ OnDemand } \\
\hline & Obs 1 & Obs 3 & Obs 1 & Obs 3 & Obs 1 & Obs 3 & Obs 1 & Obs 3 \\
\hline Mean & 2.93 & 2.94 & 2.87 & 2.65 & 3.26 & 2.71 & 3.38 & 2.90 \\
\hline SD & 1.45 & 1.52 & 1.81 & 1.52 & 1.70 & 1.38 & 1.75 & 1.31 \\
\hline $\mathrm{DE}$ & \multicolumn{2}{|c|}{1.087} & \multicolumn{2}{|c|}{1.041} & \multicolumn{2}{|c|}{0.944} & \multicolumn{2}{|c|}{1.013} \\
\hline RDE & \multicolumn{2}{|c|}{$37.0 \%$} & \multicolumn{2}{|c|}{$37.7 \%$} & \multicolumn{2}{|c|}{$31.7 \%$} & \multicolumn{2}{|c|}{$32.3 \%$} \\
\hline ICC & \multicolumn{2}{|c|}{0.625} & \multicolumn{2}{|c|}{0.759} & \multicolumn{2}{|c|}{0.786} & \multicolumn{2}{|c|}{0.738} \\
\hline 95\%confidence lower limits & \multicolumn{2}{|c|}{0.298} & \multicolumn{2}{|c|}{0.572} & \multicolumn{2}{|c|}{0.585} & \multicolumn{2}{|c|}{0.527} \\
\hline 95\%confidence Upper limits & \multicolumn{2}{|c|}{0.799} & \multicolumn{2}{|c|}{0.865} & \multicolumn{2}{|c|}{0.885} & \multicolumn{2}{|c|}{0.854} \\
\hline LOA Range & \multicolumn{2}{|c|}{0.502} & \multicolumn{2}{|c|}{0.293} & \multicolumn{2}{|c|}{0.300} & \multicolumn{2}{|c|}{0.327} \\
\hline
\end{tabular}


TABLE (4) Inter-observer reliability between observers 2 and 3

\begin{tabular}{|c|c|c|c|c|c|c|c|c|}
\hline & \multicolumn{2}{|c|}{ Dolphin side by side } & \multicolumn{2}{|c|}{ Invivo point } & \multicolumn{2}{|c|}{ Invivo voluome } & \multicolumn{2}{|c|}{ OnDemand } \\
\hline & Obs 2 & Obs 3 & Obs 2 & Obs 3 & Obs 2 & Obs 3 & Obs 2 & Obs 3 \\
\hline Mean & 3.10 & 2.94 & 3.12 & 2.65 & 3.21 & 2.71 & 3.04 & 2.90 \\
\hline SD & 1.64 & 1.52 & 1.39 & 1.52 & 1.73 & 1.38 & 1.32 & 1.31 \\
\hline $\mathrm{DE}$ & \multicolumn{2}{|c|}{0.930} & \multicolumn{2}{|c|}{0.856} & \multicolumn{2}{|c|}{1.016} & \multicolumn{2}{|c|}{0.796} \\
\hline $\mathrm{RDE}$ & \multicolumn{2}{|c|}{$30.8 \%$} & \multicolumn{2}{|c|}{$29.6 \%$} & \multicolumn{2}{|c|}{$34.3 \%$} & \multicolumn{2}{|c|}{$26.8 \%$} \\
\hline ICC & \multicolumn{2}{|c|}{0.790} & \multicolumn{2}{|c|}{0.802} & \multicolumn{2}{|c|}{0.746} & \multicolumn{2}{|c|}{0.773} \\
\hline $95 \%$ confidence lower limits & \multicolumn{2}{|c|}{0.609} & \multicolumn{2}{|c|}{0.624} & \multicolumn{2}{|c|}{0.537} & \multicolumn{2}{|c|}{0.597} \\
\hline 95\%confidence Upper limits & \multicolumn{2}{|c|}{0.887} & \multicolumn{2}{|c|}{0.893} & \multicolumn{2}{|c|}{0.860} & \multicolumn{2}{|c|}{0.873} \\
\hline LOA Range & \multicolumn{2}{|c|}{0.277} & \multicolumn{2}{|c|}{0.269} & \multicolumn{2}{|c|}{0.323} & \multicolumn{2}{|c|}{0.276} \\
\hline
\end{tabular}

\section{DISCUSSION}

CBCT superimposition is now widely applicable in dentistry. In clinical practice, analysis of 3D superimposed models is commonly performed on 3D color coded distance map or shape corresponding mesh. However, these advanced tools depend on the availability of the software and may necessitate extra fees for activation. For the sake of more applicable and less complicated analysis of superimposition, we aimed to investigate the basic linear measurement tool, and to detect to what extent it can be useful in analysis. To our knowledge, no previous studies investigated the accuracy and the reliability of $2 \mathrm{D}$ cuts of CBCT superimposition.

The participants included in this study were 21 non-growing patients (above 18 years) because we used reference landmarks that should be stable throughout the treatment span and not affected by growth. Arat et al ${ }^{[1]}$ evaluated the growth changes during puberty using lateral cephalometric superimposition and confirmed that all the cephalometric landmarks were displaced significantly due to growth.

Accuracy is best demonstrated by comparing results to a well-known gold standard. ${ }^{[2]}$ Automatic measurements of each distance on the traced CBCT image were chosen as a gold standard. The CBCT craniometric measurements were previously proved to be accurate and can be used as an orthodontic diagnostic tool. [3] Moreover, CBCT linear measurements are not affected by changes of head position which is considered a great advantage over 2D cephalometric imaging. ${ }^{[4]}$

To standardize the measurements of the gold standard, 3D cephalometric tracing was performed. In our research the 3D coordinate system for CBCT tracing of the gold standard was set to be Frankfort horizontal plane perpendicular to midsagittal plane as suggested by Balachandran et al ${ }^{[5]}$ as a common coordinate system for 3D analysis.

Dental changes were evaluated at three points; maxillary first molar, second premolar and canine. The gold standard was measured unilaterally from mid sagittal plane to compare with unilateral difference on 2D superimposed axial image. However, Cevidenes et al ${ }^{[6]}$ evaluated the CBCT superimposed models of orthognathic surgery using only inter-observer reliability between three observers. Also, Cevidenes et al ${ }^{[7]}$ didn't offer gold standard in evaluation of mandibular changes after orthognathic surgery using colour coded distance map of CBCT fused models.

We used point based and voxel-based superimposition methods to test the analysis method regardless the type of the superimposition. Four reference points were traced bilaterally; Nasion, Basion, Porion and Orbitale points. Hwang et al ${ }^{[8]}$ used $\mathrm{Na}$ (Nasion), S (Sella), Ba (Basion) and MLWS 
(Midway of lesser wing of sphenoid) to form the 3D coordinate system for registration and noted that $\mathrm{Na}, \mathrm{S}$ and $\mathrm{Ba}$ showed good reproducibility when relocated. However, Lagravere et al ${ }^{[9]}$ used AEM (Auditory external meatus) bilaterally, DFM (Dorsum foramen magnum) and ELSA (Midpoint between line connecting both foramen spinosum landmarks) and reported high sensitivity using AMIRA software (Mercury Computer Systems, Berlin, Germany).

For voxel-based method, the whole cranial base was selected to be the reference landmark for VBR as described by Cevidanes et al ${ }^{[6]}$ who used the cranial base as a stable area for non-growing cases and the anterior cranial base for growing patients. For a regional registration of a small field of view Lee et al ${ }^{[10]}$ and Koerich et al [11] described the zygomatic process of maxilla and the palate as maxillary fixed areas and symphysis and ramus as fixed regions for mandibular registration. Likewise, VBR was performed on cranial base by Motta et al ${ }^{[12]}$ using Imagine software in evaluation of orthognathic surgical outcome. Nada et al ${ }^{[13]}$ compared registration on anterior cranial base versus registration on Zygomatic arch using Maxillim software (Medicim NV, Mechelen, Belgium) and concluded that both registration areas were accepted for VBR. Koerich et al ${ }^{[11]}$ described VBR as a perfect superimposition method when cranial base structures are used as a fixed area for registration.

This study tested the accuracy of the used superimposition methods by assessment of agreement between each one with the gold standard. The superior accuracy of In Vivo volume method can be attributed to the high image quality and the automatic adjustment of the fusion process in VBR, yet it still has high relative Dahlberg error $24.7 \%$ compared to the gold standard. On the other hand, On Demand software showed the least accurate results despite being VBR method. We referred that to the poor image quality of the fusion layout that may affect the accurate delineation of margins which is expected to be better in 3D assessment using 3D colour coded distance map. Opposingly, park et al ${ }^{[14]}$ mentioned that VBR using OnDemand was an accurate and effective tool for evaluation of condylar morphological changes after bimaxillary surgery.

The decreased accuracy of PBR was attributed to the difficulty in locating the points for registration. Another study performed by Ludlow et al [15] concluded that detection of cephalometric landmarks on CBCT images was difficult and variable due to inadequate definition of the landmarks on the three dimensions. Likewise, Hwang et al ${ }^{[8]}$ thought that locating cephalometric landmarks is more liable to error in $3 \mathrm{D}$ cephalometry than $2 \mathrm{D}$ that's why $3 \mathrm{D}$ PBR is not widely used in their opinion. Cevidanes et al ${ }^{[16]}$ described the PBR as a time-consuming method that was not suitable for daily dental practice and locating several points on 3D images is more complex process than 2D lateral cephalometry.

We reported average of $4 \mathrm{~mm}$ error with assessment on 2D cut, on the contrary, Weissheimer et al ${ }^{[17]}$ quantified the superimposition error from colour coded distance map of On Demand in evaluation of orthognathic surgery was less than $0.25 \mathrm{~mm}$. we attributed the high relative error (mean 24\%) of all the tested methods to the multidirectional change in transverse, vertical, anteroposterior and possible tipping with RME as mentioned by Lagravere et al ${ }^{[18]}$ that cannot be accurately measured without information about the direction or the volume of the change on the 2D axial cut. Carvalho et al ${ }^{[19]}$ evaluated the outcome of the orthognathic surgery 1 year post operatively using 3D colour coded distance map and confirmed that the assessment would not be accurate without 3D map. Nonetheless, colour coded distance map can be a source of quantification error with incorrect outlining of the contours on the map as mentioned by Cevidanes et al. ${ }^{[16]}$

Concerning inter-observer reliability, results were inconsistent between the observers. Even 
though, the ICC of In Vivo volume was excellent (0.9), the RDE was still greater than $20 \%$. However, variability between observers ranged from $0.1 \mathrm{~mm}$ to $0.5 \mathrm{~mm}$ (LOA) that is negligible clinically and almost attributed to human error in the manual assessment. Likewise, Cevidanes et al ${ }^{[20]}$ used Imagine software VBR for registration of segmented surface models of growing cases and reported that inter-examiner range of variation was equal or less than $0.5 \mathrm{~mm}$. Koriesh et al ${ }^{[11]}$ as well used maxillary and mandibular VBR using OnDemand then reported excellent reproducibility of the results. Almukhtar et al ${ }^{[21]}$ compared VBR to SBR and reported lower variability of results with VBR with low SD and referred that due to dependence on voxel's grey scale intensity not affected by surface features resulting in more accurate representation of soft tissue changes of VBR. However, they reported no clinically significant effect. Moreover, Lee et al ${ }^{[11]}$ confirmed that VBR of OnDemand is an accurate and reliable method for evaluation of surgical treatment outcome with titanium markers on dry skulls.

The main goal of the study was to investigate, to what extent does 2D assessment using axial plane of the 3D superimposed models help in analysis, how far is it accurate and could it replace the color-coded distance map or not. From our results, we reported high relative error of all methods and inconsistent reliability of the 2D assessment using axial plane of 3D superimposed models. However, the reported error was clinically negligible, and this method can be sufficient in clinical practice. In evaluation of superimposed models especially cases of RME, the change is multidirectional thus axial plane didn't provide information about the volume or the direction of the change. We suggest that, the results would differ in more simple conditions such as bone graft analysis or healing of surgical defects. Finally, the image quality provided by the software program of the superimposed models was a main controlling factor for delineation of the borders, consequently, accuracy of the results may be affected.

\section{CONCLUSION}

The linear measurements on axial plane of CBCT superimposed models showed $3 \mathrm{~mm}$ average error. This indicates that, the statistically high relative error is clinically accepted. However, it is not recommended to depend on linear measurements alone for analysis. So, from our results, we reported weak accuracy of the linear measurements on axial plane of CBCT superimposed models and not recommending it alone for analysis.

\section{Abbreviations (in alphabetical order)}

2D: Two dimensional

3D: Three dimensional

AEM: Auditory external meatus

Ba: Basion

CBCT: Cone beam computed tomography

CEJ: Cemento-enamel junction

DBM: Data base management

DE: Dahlberg error

DFM: Dorsum foramen magnum

DICOM: Digital Imaging and Communications in Medicine

ELSA: Midpoint between line connecting both foramen spinosum landmarks)

FOV: Field of view

ICC: intra-class correlation coefficient

Kvp: Kilo voltage peak

LOA: Limit of agreement

MLWS: Midway of lesser wing of sphenoid

MPR: Multiplanar reformation

Na: Nasion

PBR: Point based registration

RDE: Relative Dahlberg error

RME: Rapid maxillary expansion 


\section{S: Sella}

VBR: Voxel based registration

VOI: Volume of interest

\section{REFERENCES}

1. Arat ZM, Rübendüz M, Arman Akgül A. The displacement of craniofacial reference landmarks during puberty: a comparison of three superimposition methods. The Angle orthodontist. 2003 Aug;73(4):374-80.

2. BS ISO 5725-1: "Accuracy (trueness and precision) of measurement methods and results - Part 1: General principles and definitions.”, p.1 (1994)

3. Gribel BF, Gribel MN, Frazão DC, McNamara Jr JA, Manzi FR. Accuracy and reliability of craniometric measurements on lateral cephalometry and 3D measurements on CBCT scans. The Angle orthodontist. 2011 Jan;81(1):2635 66.

4. El-Beialy AR, Fayed MS, El-Bialy AM, Mostafa YA. Accuracy and reliability of cone-beam computed tomography measurements: Influence of head orientation. American Journal of Orthodontics and Dentofacial Orthopedics. 2011 Aug 31;140(2):157-65. 67.

5. Balachandran R, Kharbanda OP, Gupta A. Common 3-dimensional coordinate system for assessment of directional changes. American Journal of Orthodontics and Dentofacial Orthopedics. 2016 Sep 1;150(3):398.

6. Cevidanes LH, Bailey LJ, Tucker GR, Styner MA, Mol A, Phillips CL, Proffit WR, Turvey T. Superimposition of 3D cone-beam CT models of orthognathic surgery patients. Dento maxillo facial radiology. 2005 Nov;34(6):369-75

7. Cevidanes LH, L'Tanya JB, Tucker SF, Styner MA, Mol A, Phillips CL, Proffit WR, Turvey T. Three-dimensional cone-beam computed tomography for assessment of mandibular changes after orthognathic surgery. American journal of orthodontics and dentofacial orthopedics. 2007 Jan 31;131(1):4450.

8. Hwang JJ, Kim KD, Park H, Park CS, Jeong HG. Factors Influencing Superimposition Error of 3D Cephalometric Landmarks by Plane Orientation Method Using 4 Reference Points: 4 Point Superimposition Error Regression Model. PloS one. 2014 Nov 5;9(11):e110665.

9. Lagravere MO, Major PW, Carey J. Sensitivity analysis for plane orientation in three-dimensional cephalometric analysis based on superimposition of serial cone beam computed tomography images. Dentomaxillofacial Radiology. 2014 Jan 28.

10. Lee Y, Chun YS, Kang N, Kim M. Volumetric changes in the upper airway after bimaxillary surgery for skeletal class III malocclusions: a case series study using 3-dimensional cone-beam computed tomography. Journal of Oral and Maxillofacial Surgery. 2012 Dec 31;70(12):2867-75

11. Koerich L, Burns D, Weissheimer A, Claus JD. Threedimensional maxillary and mandibular regional superimposition using cone beam computed tomography: a validation study. International journal of oral and maxillofacial surgery. 2016 May 31;45(5):662-9.

12. Motta AT, Carvalho FD, Oliveira AE, Cevidanes LH, Almeida MA. Superimposition of 3D cone-beam CT models in orthognathic surgery. Dental press journal of orthodontics. 2010 Apr;15(2):39-41.

13. Nada RM, Maal TJ, Breuning KH, Berge SJ, Mostafa YA, Kuijpers-Jagtman AM. Accuracy and reproducibility of voxel based superimposition of cone beam computed tomography models on the anterior cranial base and the zygomatic arches. PLoS One. 2011 Feb 9;6(2):e16520.

14. Park SB, Yang YM, Kim YI, Cho BH, Jung YH, Hwang DS. Effect of bimaxillary surgery on adaptive condylar head remodeling: metric analysis and image interpretation using cone-beam computed tomography volume superimposition. J Oral Maxillofac Surg. 2012; 70:1951-9.

15. Ludlow JB, Gubler M, Cevidanes L, Mol A. Precision of cephalometric landmark identification: cone-beam computed tomography vs conventional cephalometric views. Am J Orthod Dentofacial Orthop. 2009;136:312

16. Cevidanes LH, Oliveira AE, Grauer D, Styner M, Proffit WR. Clinical application of 3D imaging for assessment of treatment outcomes. InSeminars in orthodontics $2011 \mathrm{Mar}$ 31 (Vol. 17, No. 1, pp. 72-80). WB Saunders.

17. Weissheimer A, Menezes LM, Koerich L, Pham J, Cevidanes LH. Fast threedimensional superimposition of cone beam computed tomography for orthopaedics and orthognathic surgery evaluation. International journal of oral and maxillofacial surgery. 2015 Sep 30;44(9):1188-96.

18. Lagravère MO, Carey J, Heo G, Toogood RW, Major PW. Transverse, vertical, and anteroposterior changes from bone-anchored maxillary expansion vs traditional rapid maxillary expansion: a randomized clinical trial. American Journal of Orthodontics and Dentofacial Orthopedics. 2010 Mar 31;137(3):304-e1 
19. Carvalho FD, Cevidanes LH, da Motta AT, de Oliveira Almeida MA, Phillips C. Three-dimensional assessment of mandibular advancement 1 year after surgery. American Journal of Orthodontics and Dentofacial Orthopedics. 2010 Apr 30;137(4):S53-e1.

20. Cevidanes LH, Heymann G, Cornelis MA, DeClerck HJ, Tulloch JC. Superimposition of 3-dimensional conebeam computed tomography models of growing patients.
American Journal of Orthodontics and Dentofacial Orthopedics. 2009 Jul 31;136(1):94-9.

21. Almukhtar A, Ju X, Khambay B, McDonald J, Ayoub A. Comparison of the accuracy of voxel based registration and surface based registration for 3D assessment of surgical change following orthognathic surgery. PloS one. 2014 Apr 2;9(4): e9340 